\title{
REDE GEODÉSICA PARA O MONITORAMENTO COSTEIRO DO LITORAL SETENTRIONAL DO ESTADO DO RIO GRANDE DO NORTE
}

\author{
Geodetic Network for Coastal Monitoring of Setentrional Littoral of Rio Grande do \\ Norte State \\ MARCELO SOARES TELES SANTOS \\ VENERANDO EUSTÁQUIO AMARO \\ Universidade Federal do Rio Grande do Norte - UFRN \\ DG/UFRN - Departamento de Geologia \\ mstsantos@yahoo.com.br
}

\begin{abstract}
Este trabalho apresenta os procedimentos técnicos envolvidos na implantação da Rede GPS do Litoral Setentrional do Rio Grande do Norte (RGLS), onde o objetivo é fornecer subsídios fundamentais aos levantamentos geodésicos destinados ao monitoramento de áreas costeiras, submetidas à intensa dinâmica e de grande importância socioeconômica e ecológica para o Estado do Rio Grande do Norte. A metodologia permitiu a determinação das coordenadas geodésicas e altitudes ortométricas das estações com precisão decimétrica em relação ao Sistema Geodésico Brasileiro (SGB), a partir do método de posicionamento relativo e da altimetria por GPS. Ainda, foi possível o estudo de aspectos geodésicos envolvidos na altimetria por GPS, tais como avaliação da situação física e da densidade das Referências de Nível (RRNN) disponíveis, avaliação absoluta e relativa do modelo geoidal, proposição de metodologia para a altimetria por GPS de precisão e desenvolvimento de software para essa finalidade, que contribuíram para o conhecimento geodésico na área de estudo.
\end{abstract}

Palavras chave: Rede Geodésica; GPS; Monitoramento Costeiro.

ABSTRACT
This paper presents the techniques procedures involved in implantation of the RGLS (Rede GPS do Litoral Setentrional do Rio Grande do Norte), with the objective of serving basic subsidies for geodetic surveys for monitoring of coastal areas, subject to intense geodynamic activity and socio-economic and ecological importance to the 
Rio Grande do Norte State. The methodology allowed the determination of geodetic coordinates and orthometric heights of stations with decimeters precision concerning the Brazilian Geodetic System (BGS), from positioning and leveling by GPS on relative method. In addition, it was possible the study of geodetic aspects involving GPS altimetry such as evaluation of the physical situation and the density of Benchmarks available, absolute and relative evaluation of the geoid model, the proposition of a methodology for precision GPS altimetry and development of a software for this purpose which contributed to geodetic knowledge in the studied area.

Keywords: Geodetic Network; GPS; Coastal Monitoring.

\section{INTRODUÇÃO}

Classicamente, o monitoramento terrestre de zonas costeiras era realizado a partir de perfis topográficos dispostos perpendicularmente à Linha de Costa (LC) e sobre os quais eram calculados os recuos ou avanços das LC, com o objetivo de identificar áreas de acresção ou erosão. No entanto, tais levantamentos somente forneciam informações pontuais e locais da dinâmica das praias, muitas vezes não sendo representativos da complexidade da dinâmica costeira e insuficientes para o cálculo de áreas e volumes de erosão ou acresção (CHAVES, 2005; LIMA, 2004). Com o avanço e proliferação das técnicas espaciais de posicionamento, especialmente o GPS (Global Positioning System), o método clássico foi substituído pelo espacial, com os benefícios da precisão, simplicidade operacional, rapidez e baixo custo (SANTOS, 2011; TANAJURA et al., 2011; GONÇALVES et al., 2010; ROCHA et al., 2009; BAPTISTA et al., 2008). A partir dessa técnica, cada vez mais precisa e rápida, tornou-se possível a modelagem tridimensional de precisão de extensas áreas costeiras em um reduzido intervalo de tempo. A comparação dos Modelos Digitais de Elevação (MDE) georreferenciados obtidos sistematicamente ao longo do tempo permite, além da identificação e da mensuração da variação da LC e do cálculo das áreas de erosão ou acresção, o cálculo do volume e da orientação do transporte de sedimentos.

No entanto, para o monitoramento de extensas áreas costeiras em curto intervalo de tempo e com precisão, devem ser adotados procedimentos adequados de aquisição e processamento dos dados GPS. A aquisição pode ser feita com o posicionamento relativo cinemático (em tempo real ou pós-processado), no qual, as coordenadas dos pontos de interesse são determinadas com sessões de observações de curtas durações, o que requer a utilização de bases curtas para se obter precisão. Nesse caso, o levantamento de extensas áreas costeiras, da ordem de dezenas de quilômetros, requer a criação de uma infra-estrutura geodésica de referência para fornecer bases curtas em toda a área a ser levantada, além da aplicação de uma logística de campo eficiente que possibilite os citados benefícios do posicionamento GPS. Tal infra-estrutura consiste em estações geodésicas implantadas ao longo da área de estudo, posicionadas com precisão e materializadas de forma definitiva, as 
quais devem possuir coordenadas e altitudes conhecidas, servindo de referência aos levantamentos planialtimétricos do GPS.

Na área de estudo, localizada no Litoral Setentrional do Rio Grande do Norte (RGLS), o monitoramento costeiro terrestre tem sido realizado pelo método de posicionamento relativo cinemático do GPS a partir da Rede GPS do Litoral Setentrional do Rio Grande do Norte (RGLS), implantada para dar apoio básico aos levantamentos geodésicos regionais de monitoramento costeiro na área, planimétricos e altimétricos. As coordenadas geodésicas e as altitudes ortométricas das estações da rede foram determinadas com precisão decimétrica em relação ao Sistema Geodésico Brasileiro (SGB): as coordenadas foram determinadas pelo método de posicionamento relativo estático, usando estações da Rede Brasileira de Monitoramento Contínuo (RBMC) como estações base para o processamento dos dados; as altitudes ortométricas foram calculadas pela altimetria por GPS no método relativo, usando Referências de Nível (RRNN) da Rede Altimétrica Fundamental do Brasil (RAFB) e o modelo geoidal do Instituto Brasileiro de Geografia e Estatística (IBGE), modelo MAPGEO2004. Na determinação das coordenadas e altitudes das estações da rede, foram desenvolvidas as seguintes atividades: avaliação da situação física e da densidade dos marcos geodésicos de RRNN disponíveis, avaliação absoluta e relativa do modelo geoidal, importante na altimetria por GPS; proposição de metodologia para a altimetria por GPS de precisão; e desenvolvimento de aplicativo para o cálculo das altitudes ortométricas a partir do posicionamento por GPS. Portanto, este trabalho teve o objetivo geral de apresentar os dados, a metodologia e os resultados obtidos na implantação da RGLS, que consistiu no estudo e materialização de uma estrutura geodésica de referência aos futuros levantamentos a serem executados em toda a área de abrangência da rede.

Devido aos citados benefícios, o GPS já foi utilizado e avaliado em monitoramentos costeiros em algumas partes do mundo (SANTOS, 2011; TANAJURA et al., 2011; GONÇALVES et al., 2010; ROCHA et al., 2009; BAPTISTA et al., 2008). O grande problema encontrado nas diversas metodologias aplicadas está na adoção de diferentes referenciais geodésicos e indicadores de LC, o que dificulta ou inviabiliza a comparação dos resultados de pesquisas realizadas em épocas diferentes (domínio temporal), mesmo em uma mesma área (domínio espacial). Uma rede geodésica como a implantada, com referencial geodésico unívoco, fixo e relativamente estável no tempo, que forneça estações com coordenadas e altitudes de precisão e que seja materializada de maneira definitiva seria suficiente para resolver o problema da multiplicidade dos referenciais. Dessa forma, todos os levantamentos, presentes, passados e futuros realizados na área de abrangência da rede poderiam ser relacionados sempre ao mesmo referencial, permitindo a analise multitemporal de precisão dos elementos de interesse. Ainda, por utilizar a estrutura geodésica nacional oficial em sua implantação, os levantamentos podem ser convertidos para qualquer outro referencial geodésico do mundo, o que permite a conversão e a comparação de levantamentos realizados em outras regiões. 


\section{2. ÁREA DE ESTUDO E ESTAÇÕES DA RGLS}

A área de estudo, composta por um sistema de praias oceânicas, ilhas barreiras e canais de maré, está localizada no Litoral Setentrional do Estado do Rio Grande do Norte (RN), entre os municípios de Macau e Guamaré, numa faixa de aproximadamente $30 \mathrm{~km}$ de litoral. A Figura 1 apresenta o mapa geomorfológico da área de estudo com a distribuição espacial dos 4 trechos de abrangência da RGLS, a Praia da Soledade, a Ilha Ponta do Tubarão, a Ilha do Fernandez e a Praia do Minhoto, nos quais foram implantadas as nove estações da RGLS em destaque. As estações foram distribuídas próximas aos principais trechos de interesse para monitoramentos costeiros, ou seja, dunas móveis e zonas de praia. A área é de grande importância ecológica e econômica para o Estado do RN, tendo sido objeto de trabalhos sobre monitoramento costeiro, principalmente com o uso de imagens de satélites (SOUTO et al., 2004; GRIGIO et al., 2005). Nela está contida a Reserva de Desenvolvimento Sustentável Ponta do Tubarão (RDSPT) e atividades antrópicas importantes para o Estado, destacando-se os pólos industriais petrolífero, salineiro e de carcinicultura. É caracterizada pela intensa ação de processos costeiros, tais como transporte eólico e litorâneo, erosão generalizada, alterações no balanço de sedimentos, variações na LC, abertura e fechamento de canais de marés e formação de ilhas barreiras. A intensa dinâmica tem provocado sérios problemas às atividades socioeconômicas presentes na área, além de se configurar como um potencial problema ambiental (SOUTO et al., 2004; GRIGIO et al., 2005).

Figura 1 - Mapa geomorfológico da área de estudo (Silva et al., 2010), com a distribuição espacial dos 4 trechos selecionados para o monitoramento (Praia de Soledade, Ilha da Ponta do Tubarão, Ilha do Fernandez, e Praia de Minhoto) e das 9

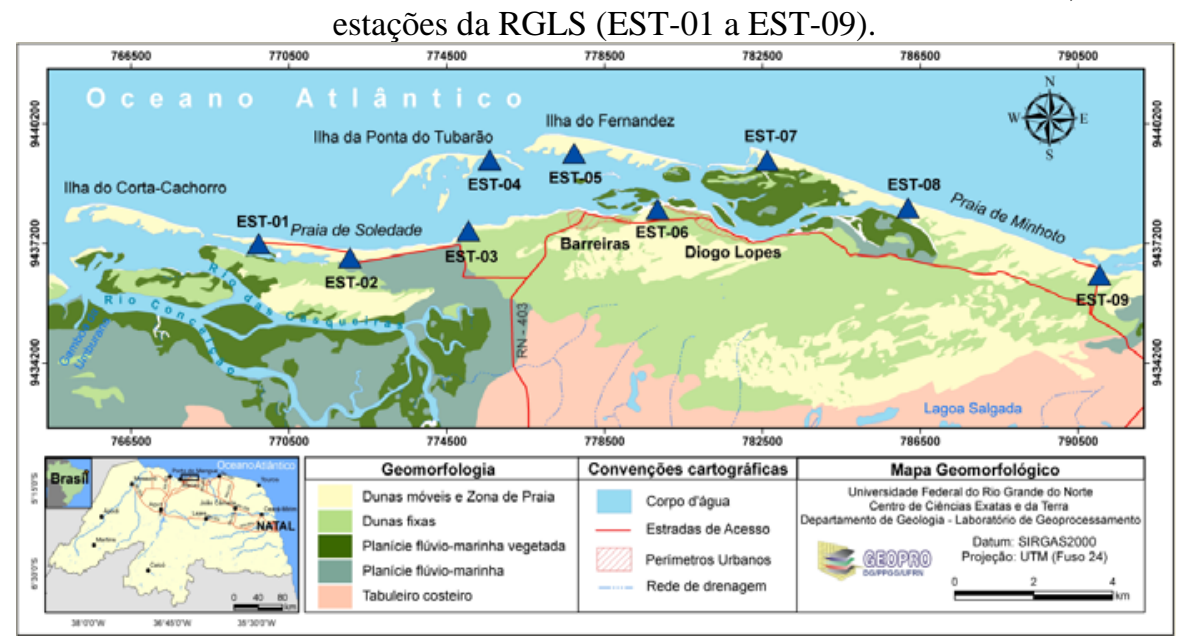

Bol. Ciênc. Geod., sec. Artigos, Curitiba, v. 17, no 4, p.571-585, out-dez, 2011. 


\section{REDE GPS, REDE ALTIMÉTRICA E MODELO GEOIDAL DE REFERÊNCIA}

Para a adequada utilização do GPS na determinação de coordenadas geodésicas e altitudes ortométricas, faz-se necessário o uso de dados que possibilitem o emprego desta técnica de maneira eficiente e precisa. Na determinação das coordenadas geodésicas, é necessária uma rede geodésica que forneça estações de referência para o posicionamento relativo GPS. Na determinação da altitude ortométrica, deve-se utilizar Referências de Nível (RRNN) e um modelo geoidal. Neste item descrevem-se os dados utilizados na determinação das coordenadas geodésicas e altitudes ortométricas das estações da RGLS, consistindo de três classes: rede GPS de referência, rede altimétrica de referência e modelo geoidal.

A Rede Brasileira de Monitoramento Contínuo (RBMC) do GNSS (Global Navigation Satellite System), implantada e mantida pelo IBGE, foi utilizada como referência na determinação das coordenadas geodésicas das estações da RGLS. A RBMC é a rede de referência ativa adotada oficialmente no Brasil e possui, atualmente, 72 estações operacionais. Além de fornecer coordenadas precisas das estações, a RBMC coleta observações dos satélites GNSS continuamente nas duas frequências (L1 e L2) e disponibiliza-os alguns dias após a coleta. Isso possibilita a qualquer usuário, com apenas um receptor, realizar o posicionamento relativo. Os arquivos das sessões de observações de cada estação da RBMC, com duração de 24 horas, são disponibilizados no formato RINEX (Receiver INdependent data EXchange format), via internet, na página do IBGE (IBGE, 2010). Os arquivos RINEX possuem formato padrão, podendo ser utilizados por qualquer programa de processamento GNSS.

A Rede Altimétrica Fundamental do Brasil (RAFB), implantadas pelo IBGE, forneceu as Referências de Nível (RRNN) utilizadas na altimetria por GPS das estações da RGLS. Elas foram implantadas em linhas de nivelamento geométrico desde a década de 40 , as quais seguiram, tanto quanto possível, as melhores estradas de rodagem e, em algumas regiões, as estradas de ferro. As altitudes das RRNN que compõe a RAFB são referidas ao nível médio do mar, dado pelo marégrafo de Imbituba, no Estado de Santa Catarina. As RRNN, implantadas com espaçamento médio de $5 \mathrm{~km}$, são materializadas por marcos de concreto e identificadas com chapas de bronze. Também são usadas chapas metálicas, chumbadas em monumentos, soleiras de igrejas, plataformas de estações ferroviárias, pontes, etc. A localização e os dados das estações são obtidos em suas monografias, no Banco de Dados Geodésicos do IBGE, disponível no site (IBGE, 2010).

O modelo geoidal utilizado na altimetria por GPS das estações da RGLS foi o modelo MAPGEO2004, desenvolvido e disponibilizado pelo IBGE em conjunto com diversas instituições do Brasil. O modelo, que possui resolução espacial de 5' de arco, foi determinado pela fórmula de Stokes, usando dados do modelo geopotencial EGM96, dados gravimétricos terrestres e oceânicos e dados topográficos na forma digital. As alturas geoidais são calculadas no programa de 
forma relativamente simples a partir da inserção das coordenadas geodésicas dos pontos, em SAD69 ou SIRGAS2000. Ele é disponibilizado gratuitamente no site do IBGE (IBGE, 2010).

\section{METODOLOGIA EXPERIMENTAL}

Este capítulo apresenta os critérios utilizados na seleção, materialização e posicionamento planialtimétrico das estações da RGLS.

\subsection{Seleção de Estações da RGLS}

A distribuição geográfica das estações foi definida com o objetivo de proporcionar distancias curtas entre os pontos definidores das LC e as estações da rede em toda a extensão da área, para permitir alta precisão ao posicionamento relativo cinemático do GPS. A distância máxima de 3,5 km ocorre no trecho da Ilha Ponta do Tubarão. Como se pretende o monitoramento da zona costeira em séries temporais intra anuais e interanuais, todas as estações foram implantadas em locais de fácil acesso e sem obstáculos que interfiram na recepção dos sinais dos satélites (edificações, vegetação, etc.). Ainda, todas as estações da rede foram materializadas e documentadas. A materialização foi feita com marcos de concreto, com dimensões de 20x40x60 cm, identificados com plaquetas de metal do GEOPRO/DG/UFRN. A documentação foi feita através das monografias dos marcos geodésicos, que contém uma síntese dos dados de identificação (nome, número, coordenadas e altitudes), um croqui com o local da estação, um roteiro para acesso à estação, e fotografias com a localização e o detalhe da estação.

\subsection{Determinação das Coordenadas Geodésicas}

A aquisição de dados foi feita com o posicionamento GPS pelo método relativo estático, em duas etapas. A primeira etapa consistiu no posicionamento da estação EST-06, localizada no centro da área. Para isso, foram usadas, como referências, as duas estações mais próximas da RBMC, Natal (RNNA) e Mossoró (RNMO), distantes, respectivamente, de $95 \mathrm{~km}$ e $164 \mathrm{~km}$ da EST-06. Devido às grandes distâncias entre as estações de referência e a EST-06, a duração da sessão foi de seis horas. A segunda etapa consistiu no posicionamento das demais estações da rede, com uso da EST-06 como referência. Essa estratégia permitiu o uso de distâncias curtas entre a estação de referência e as demais estações, possibilitando o uso de sessões com durações de apenas uma hora. Como o posicionamento da EST06 foi feito a partir das estações da RBMC, toda a rede GPS foi georreferenciada ao Sistema Geodésico Brasileiro (SGB), já no sistema SIRGAS2000. Para aumentar a confiabilidade aos resultados obtidos, a estação EST-06 e as demais estações da rede foram determinadas em duas sessões de observações distintas. Assim, após o processamento das sessões, as coordenadas e as precisões de cada estação da rede foram determinadas, respectivamente, pela média e desvio padrão amostrais. As sessões foram realizadas em três dias consecutivos, em maio de 2010, e envolveram receptores GPS de dupla e de simples frequência. O receptor utilizado na estação 
EST-06 foi do modelo Trimble 5700 e os da RBMC foram dos modelos Trimble 4000 SSI e Leica GRS 1200, todos de dupla frequência. O receptor utilizado nas estações da rede foi do modelo Trimble R3, de simples frequência, que tem precisão nominal de $\pm 5 \mathrm{~mm}+0,5 \mathrm{ppm}$ na horizontal e $\pm 5 \mathrm{~mm}+1 \mathrm{ppm}$ na vertical. Nos processamentos e ajustamentos de dados GPS, efetuados com o programa Topcon Tools versão 6.04, foram usadas as efemérides precisas e as observações da fase da onda portadora (L1/L2 no processamento da estação EST-06 e L1 nas demais estações). No processamento da estação EST-06, por envolver vetor maior do que $100 \mathrm{~km}$, foi gerada a solução livre da ionosfera; nos processamentos das demais estações da rede, por envolverem vetores curtos menores do que $12 \mathrm{~km}$, a única solução admitida foi a fixa (fixação das ambigüidades como inteiros), por garantir precisão e confiabilidade ao processamento, como em Tanajura (2011). Mais detalhes sobre os métodos de aquisição e processamento de dados GPS podem ser encontrados em Monico (2007) e Seeber (2003).

\subsection{Determinação das Altitudes Ortométricas}

As altitudes ortométricas, relacionada ao geóide (equipotencial do campo de gravidade da Terra que coincide com o nível médio dos mares), foram determinadas a partir das altitudes geométricas, obtidas por GPS e referidas ao elipsóide de revolução adotado no posicionamento.

\subsubsection{Altimetria por GPS}

A altimetria por GPS consiste em determinar a altitude ortométrica de um ponto de interesse $(i)$ em relação a, pelo menos, uma estação de referência $(A)$, de coordenadas geodésicas $\left(\alpha_{A}, \lambda_{A}, h_{A}\right)$ e altitudes ortométricas $\left(H_{A}\right)$ conhecidas. Isso é feito através das diferenças de altitudes geométricas $\left(\Delta h_{i}=h_{i}-h_{A}\right)$, determinadas pelo GPS, e das diferenças de alturas geoidais $\left(\Delta N_{i}=N_{i}-N_{A}\right)$, determinadas a partir de um modelo geoidal (Featherstone et al., 1998; Ollikainen, 1998):

$$
H_{i}=H_{A}+\Delta H_{i}=H_{A}+\left(\Delta h_{i}-\Delta N_{i}\right)
$$

Com o objetivo de aumentar a confiabilidade e estimar a precisão dos resultados, as altitudes ortométricas das estações da rede foram calculadas a partir de várias estações de referência da área de estudo. Assim, um conjunto de altitudes $\left\{H_{1}, H_{2}, H_{3}, \cdots, H_{m}\right\}$ foi obtido para cada estação da rede, a partir do qual foram calculados a média aritmética e o desvio padrão amostrais para estimar, respectivamente, a altitude da estação e sua precisão. 
4.3.2. Posicionamento GPS em Referências de Nível (RRNN)

Como referências na altimetria das estações da RGLS, foram utilizadas Referências de Nível (RRNN) pertencentes à Rede Altimétrica Fundamental do Brasil (RAFB), que possuem altitudes ortométricas conhecidas. Como as RRNN não possuem coordenadas geodésicas de precisão, estas foram determinadas com 0 posicionamento GPS. Assim, todas as RRNN passaram a ter altitude ortométrica e coordenadas geodésicas, requisitos básicos para serem utilizadas como referências na altimetria por GPS. As RRNN selecionadas estão localizadas em um raio de 25 km da área de estudo, nos municípios de Macau (RN446K, RN2411J, RN2411R) e Guamaré (RN2412B). Os equipamentos, a metodologia e os procedimentos de aquisição e processamento dos dados foram os mesmos utilizados no posicionamento das estações da RGLS (Seção 4.2). Assim, as coordenadas geodésicas da RN2411R, localizada no centro da área, foram determinadas em relação às estações mais próximas da RBMC, Natal (RNNA) e Mossoró (RNMO), com sessão de observações de 6 horas. As demais RRNN foram determinadas em relação à RN2411R, com sessão de 1 hora. As sessões foram realizadas em três dias consecutivos, em fevereiro de 2010.

\subsubsection{Avaliação do Modelo Geoidal}

Como os resultados da altimetria por GPS no método relativo estão diretamente relacionados com a acurácia das diferenças de aturas geoidais, há necessidade de avaliação do modelo geoidal, antes de seu uso, para verificar as potencialidades do método. Esta avaliação foi realizada de duas formas: absoluta, que avalia as alturas geoidais; e relativa, que avalia as diferenças de alturas geoidais.

A avaliação absoluta consistiu no cálculo das discrepâncias das alturas geoidais gravimétricas $\left(N_{g}\right)$, obtidas no modelo geoidal, em relação às alturas geoidais geométricas $\left(N_{G P S}\right)$, obtidas pelo posicionamento GPS em RRNN $\left(N_{G P S}\right)$ :

$$
\triangle N=N_{g}-N_{G P S}
$$

onde, $\Delta N$ é a discrepância da altura geoidal gravimétrica, $\mathrm{e}$

$$
N_{G P S}=h-H
$$

sendo, $h$ e $H$, respectivamente, as altitudes geométrica e ortométrica do ponto.

A avaliação relativa baseou-se no cálculo, em pares de Referências de Nível $R N_{i}$ e $R N_{j}$, das discrepâncias das diferenças de alturas geoidais gravimétricas $\left(\Delta N_{g_{i j}}=N_{g_{i}}-N_{g_{j}}\right)$, obtidas no modelo geoidal, em relação às respectivas 
diferenças de alturas geoidais geométricas $\left(\Delta N_{G P S_{i j}}=N_{G P S_{i}}-N_{G P S j}\right)$, obtidas pelo posicionamento GPS em RRNN (Kiamehr, 2002; Kotsakis \& Sideris, 1999):

$$
\delta \Delta N_{i j}=\Delta N_{g_{i j}}-\Delta N_{G P S_{i j}}
$$

onde, $\delta \Delta N_{i j}$ é a discrepância da diferença de altura geoidal gravimétrica, e

$$
\begin{aligned}
& N_{G P S_{i}}=\left(h_{i}-H_{i}\right) \\
& N_{G P S_{j}}=\left(h_{j}-H_{j}\right)
\end{aligned}
$$

sendo, $h_{i}$ e $h_{j}$ as altitudes geométricas nas estações $i$ e $j$, e $H_{i}$ e $H_{j}$ as altitudes ortométricas nas estações $i$ e $j$.

Para o conjunto de discrepâncias $\left(\varepsilon_{i}\right)$ das alturas geoidais e das diferenças de alturas geoidais, a medida de acurácia foi dada pelo erro quadrático médio (EQM), em inglês "Mean Square Error (MSE)", dado por (Monico, 2009 apud Mikhail \& Ackermann, 1976):

$$
M S E=\sqrt{\sum_{i=1}^{n} \frac{\varepsilon_{i}^{2}}{n}}
$$

\section{RESULTADOS E DISCUSSÃO}

Este capítulo apresenta e analisa as coordenadas geodésicas e as altitudes ortométricas obtidas nas campanhas de observações, propõe estratégias para a altimetria por GPS e apresenta o aplicativo desenvolvido para tal finalidade.

\subsection{Coordenadas Geodésicas das Estações da RGLS}

A Tabela 1 apresenta as coordenadas geodésicas e as respectivas precisões das estações da RGLS, obtidos com os processamentos de dados GPS. As coordenadas foram apresentadas no sistema SIRGAS2000 e projeção UTM (MC=-39). No processamento da EST-06, com uso das estações da RBMC como referências, as precisões obtidas foram de $1,6 \mathrm{~cm}$ em $\mathrm{N}, 2,8 \mathrm{~cm}$ em E e 4,5 cm em h. No processamento das demais estações da rede, com uso da estação EST-06 como referência, as precisões obtidas tiveram média de $0,4 \mathrm{~cm}$ em $\mathrm{N}, 0,5 \mathrm{~cm}$ em E e 1,0 $\mathrm{cm}$ em $\mathrm{h}$. Os resultados mostram que a EST-06, localizada no centro da área, possui precisão decimétrica em relação à RBMC, ou seja, ao Sistema Geodésico Brasileiro (SGB), e as demais estações possuem precisão centimétrica em relação à EST-06. As precisões obtidas são resultados da combinação dos diversos parâmetros básicos de aquisição e processamento de dados, tais como tipos de receptores, duração da 
sessão e comprimento da base. No posicionamento da estação EST-06, por envolver bases longas, de $95 \mathrm{~km}$ e $164 \mathrm{~km}$, foram utilizados receptores GPS de dupla frequência e duas sessões de 6 horas. No posicionamento das demais estações da rede, para as bases curtas de no máximo $12 \mathrm{~km}$, foi utilizado receptor GPS de simples frequência e duas sessões de 1 hora.

Tabela 1 - Coordenadas geodésicas das estações da RGLS.

\begin{tabular}{c|c|c|c|c|c|c}
\hline Número & $\boldsymbol{N}$ & $\boldsymbol{E}$ & $\boldsymbol{h}$ & $\boldsymbol{s} \boldsymbol{N}$ & $\boldsymbol{s} \boldsymbol{E}$ & $\boldsymbol{s h}$ \\
\hline EST06 & $\mathbf{9 4 3 8 0 5 7 , 0 8 4}$ & $\mathbf{7 7 9 8 3 8 , 9 1 6}$ & $\mathbf{1 3 , 3 1 4}$ & $\mathbf{0 , 0 1 6}$ & $\mathbf{0 , 0 2 8}$ & $\mathbf{0 , 0 4 5}$ \\
\hline EST01 & 9437196,641 & 769729,425 & $-0,929$ & 0,004 & 0,004 & 0,010 \\
\hline EST02 & 9436845,892 & 772045,951 & $-3,852$ & 0,006 & 0,007 & 0,018 \\
\hline EST03 & 9437521,423 & 775046,666 & 6,531 & 0,004 & 0,004 & 0,010 \\
\hline EST04 & 9439300,026 & 775589,141 & $-3,845$ & 0,002 & 0,002 & 0,005 \\
\hline EST05 & 9439469,370 & 777724,740 & $-3,437$ & 0,006 & 0,008 & 0,012 \\
\hline EST07 & 9439286,046 & 782618,253 & 1,713 & 0,004 & 0,005 & 0,011 \\
\hline EST08 & 9438093,202 & 786190,59 & $-2,553$ & 0,002 & 0,002 & 0,006 \\
\hline EST09 & 9436410,390 & 791045,319 & $-2,083$ & 0,004 & 0,004 & 0,010 \\
\hline & & MÉDIA & & $\mathbf{0 , 0 0 4}$ & $\mathbf{0 , 0 0 5}$ & $\mathbf{0 , 0 1 0}$ \\
\hline & & D.P. & & $\mathbf{0 , 0 0 2}$ & $\mathbf{0 , 0 0 2}$ & $\mathbf{0 , 0 0 4}$ \\
\hline
\end{tabular}

\subsection{Altitudes Ortométricas das Estações da RGLS}

No processamento da RN2411R, com uso das estações da RBMC como referências, foram obtidas coordenadas com precisão de 1,3 cm, 2,1 cm e 3,6 cm, respectivamente, nas componentes $\mathrm{E}, \mathrm{N}$ e h. No processamento das demais RRNN (RN446K, RN2411J, RN2411R, RN2412B), usando a estação RN2411R como referência, foram obtidas precisões com médias de $0,7 \mathrm{~cm}$ em N, 0,9 cm em E e 2,1 $\mathrm{cm}$ em h. De acordo com os resultados, as coordenadas das RRNN foram obtidas nas mesmas margens de erros das coordenadas das estações da RGLS, ou seja, de centímetros. Isso ocorreu devido à aplicação, nos dois casos, dos mesmos equipamentos e metodologias de aquisição e processamento dos dados.

A partir das cinco RRNN posicionadas com GPS, os erros absolutos e relativos do modelo geoidal foram calculados. Na avaliação do erro absoluto (Equação 2), as cinco RRNN forneceram cinco alturas geoidais gravimétricas, as quais, comparadas com as respectivas alturas geoidais geométricas, apresentaram discrepâncias com as seguintes estatísticas: média de $-44,0 \mathrm{~cm}$, desvio padrão de 5,3 cm, MSE de 44,3 cm, mínimo de $-51,4$ cm e máximo de $-38,0 \mathrm{~cm}$. Na avaliação do erro relativo (Equação 4), as cinco RRNN geraram dez diferenças de alturas geoidais gravimétricas, as quais, comparadas com as respectivas diferenças de alturas geoidais geométricas, apresentaram discrepâncias com as seguintes estatísticas: 
média de 6,7 cm, desvio padrão de 3,7 cm, MSE de 7,5 cm, mínimo de 1,9 $\mathrm{cm}$ e máximo de 13,4 cm. As distâncias médias entre as RRNN foram de $25 \mathrm{~km}$, com mínimo de $13 \mathrm{~km}$ e máximo de $44 \mathrm{~km}$. Em termos relativos, os erros obtidos foram de $1 \mathrm{~mm} / \mathrm{km}$ a $5 \mathrm{~mm} / \mathrm{km}$.

As discrepâncias entre as alturas geoidais gravimétricas em relação às geométricas (média de $-44 \mathrm{~cm}$ ) caracterizam a componente sistemática do geóide, relacionada a fontes de erros aleatórios e sistemáticos nas variáveis envolvidas com a altimetria por GPS (altitudes ortométrica e geométrica, altura geoidal). A existência desta componente indica que o geóide gravimétrico (determinado a partir do campo de gravidade da Terra) e o geométrico (determinado por GPS em RRNN) não são perfeitamente coincidentes, ou seja, o geóide gravimétrico é incompatível com o sistema altimétrico local. Assim, as altitudes ortométricas determinadas no modo absoluto (rearranjo da Equação 3) com uso do modelo geoidal utilizado teriam um erro médio de $-0,440 \mathrm{~m}$, desconsiderando as outras fontes de erro. No entanto, as discrepâncias entre as diferenças de alturas geoidais gravimétricas em relação ás geométricas (média de $6,7 \mathrm{~cm}$ ) indicam que as altitudes ortométricas determinadas no método relativo (Equação 1) com uso do modelo geoidal utilizado teriam um erro médio de $6,7 \mathrm{~cm}$, desconsiderando as outras fontes de erros. As diferenças de alturas geoidais possuem melhor acurácia do que as alturas geoidais absolutas porque, ao subtrair duas alturas geoidais suficientemente próximas, erros sistemáticos envolvidos nos dois pontos são removidos. Assim, quanto menor a distância entre os pontos, melhor a acurácia das diferenças de alturas geoidais, como mensurado em Santos (2011).

Portanto, as altitudes ortométricas das estações da RGLS foram calculadas com a altimetria por GPS no método relativo, através da Equação (1), aproveitando a acurácia das diferenças de alturas geoidais. A Tabela 2 mostra as altitudes ortométricas e os desvios padrões das estações da RGLS, calculadas com uso das cinco RRNN de referência posicionadas com GPS e do modelo geoidal do IBGE. O desvio padrão de $5,3 \mathrm{~cm}$ foi igual para todas as estações devido ao uso das mesmas RRNN de referência nos cálculos das altitudes dos pontos, ou seja, a dispersão foi igual para todas as estações. A precisão obtida nas altitudes ortométricas, adequada à altimetria por GPS em inúmeras aplicações, se deve ao uso de estações de referência (RRNN) próximas à área de estudo, com média de $25 \mathrm{~km}$ de distância, que proporcionou diferenças de alturas geoidais com acurácia de centímetros.

\subsection{Estratégias para a Altimetria por GPS e Aplicativo Desenvolvido}

Um usuário interessado em obter as coordenadas geodésicas e a altitude ortométrica de um ponto de interesse localizado na área de estudo (ou em suas proximidades) deve seguir a seguinte estratégia: a) selecionar e localizar a estação da rede GPS mais próxima do ponto de interesse, através das monografias dos marcos geodésicos; b) determinar as coordenadas geodésicas do ponto de interesse pelo posicionamento relativo GPS usando a estação da rede como referência; c) calcular a diferença de altitude geométrica entre a estação da rede e o ponto de 
interesse; d) calcular a diferença de altura geoidal entre os dois pontos, com o modelo geoidal do IBGE; e e) a partir da Equação (1), determinar a altitude ortométrica do ponto de interesse em relação à estação da rede GPS selecionada. Se tiver interesse por mais confiabilidade aos resultados, o usuário pode usar o aplicativo desenvolvido neste trabalho, o qual calcula a altitude ortométrica de um ponto através da média aritmética das altitudes obtidas por diversas estações da RGLS. Os dados de entrada são as variáveis utilizadas na altimetria por GPS: N, E, altitude geométrica e altura geoidal do ponto. No processamento, o programa calcula a altitude ortométrica de cada ponto posicionado por meio da seguinte metodologia: a) seleciona as $n$ estações de referência mais próximas do ponto posicionado; b) calcula a altitude ortométrica do ponto em relação a cada estação de referência, usando a Equação (1); c) calcula a altitude ortométrica sua precisão, respectivamente, pela média aritmética e o desvio padrão das altitudes determinadas. O programa foi desenvolvido em planilha do Microsoft Excel e pode ser obtido no site do GEOPRO/DG/UFRN (www.petromar.geologia.ufrn.br).

Tabela 2 - Altitudes ortométricas das estações da RGLS

\begin{tabular}{c|c|c}
\hline NÚMERO & $\mathrm{H}(\mathrm{m})$ & d.p $(\mathrm{m})$ \\
\hline EST-01 & 5,511 & 0,053 \\
\hline EST-02 & 2,598 & 0,053 \\
\hline EST-03 & 12,871 & 0,053 \\
\hline EST-04 & 2,517 & 0,053 \\
\hline EST-05 & 2,883 & 0,053 \\
\hline EST-06 & 19,564 & 0,053 \\
\hline EST-07 & 7,943 & 0,053 \\
\hline EST-08 & 3,587 & 0,053 \\
\hline EST-09 & 4,177 & 0,053 \\
\hline
\end{tabular}

\section{CONCLUSÕES}

Este trabalho teve o objetivo principal de implantar a Rede GPS do Litoral Setentrional do Rio Grande do Norte (RGLS), para servir de apoio básico aos levantamentos geodésicos necessários ao monitoramento costeiro da área de estudo, sensível ambientalmente e de intensa erosão costeira sobre as atividades industriais instaladas na zona de praia. As coordenadas geodésicas das nove estações da rede foram obtidas com o posicionamento relativo GPS, com uso de estações da RBMC como referências e receptores de simples e de dupla frequência. As altitudes ortométricas foram determinadas pela altimetria por GPS no método relativo usando, como referências, cinco RRNN posicionadas na área de estudo e o modelo geoidal do IBGE, modelo MAPGEO2004. 
Como resultados dos posicionamentos das estações da RGLS, as coordenadas geodésicas da EST-06, localizada no centro da área, foram obtidas com precisão de $1,6 \mathrm{~cm}$ em E, 2,8 cm em $\mathrm{N}$ e 4,5 cm em h em relação à RBMC do SGB, e as coordenadas das demais estações da RGLS foram obtidas com precisão de 0,4 cm em E, $0,5 \mathrm{~cm}$ em $\mathrm{N}$ e $1,0 \mathrm{~cm}$ em h em relação à estação EST-06. As altitudes ortométricas das estações da RGLS tiveram precisão de 5,3 cm em relação ao datum vertical do SGB, com uso de 5 RRNN como referências na altimetria por GPS. As cinco RRNN, além de servirem de referência na altimetria por GPS, foram utilizadas na avaliação do modelo geoidal, que forneceu erros com médias de -44 $\mathrm{cm}$ nas alturas geoidais absolutas e $6,7 \mathrm{~cm}$ nas diferenças de alturas geoidais. Os erros das alturas geoidais mostraram que a altimetria por GPS de precisão deve ser realizada no método relativo, que utiliza as diferenças de alturas geoidais e permite a determinação de altitudes ortométricas com acurácia decimétrica.

Após a implantação e documentação da rede de pontos com coordenadas geodésicas e altitudes ortométricas conhecidas, a RGLS cumpriu inicialmente com seu principal objetivo, ou seja, o apoio básico para os levantamentos geodésicos necessários ao monitoramento costeiro da área de estudo. Tal monitoramento tem sido realizado a partir da comparação entre LC e MDE obtidos em levantamentos geodésicos com intervalos trimestrais (nas quatro estações do ano) com o objetivo de medir as variações superficiais e volumétricas sazonais ocorridas na zona costeira. Os resultados obtidos vêm sendo utilizados na identificação de áreas para ações de proteção e recuperação dos setores praiais onde existem instalações industriais, sobretudo da indústria petrolífera, e ocupações antrópicas; na preservação das ilhas barreiras, necessárias à manutenção dos estuários; e na prevenção de possíveis impactos ao meio ambiente pela previsão dos picos de erosão e na identificação de necessidade de implantação de obras de contenção da erosão (SANTOS, 2011).

Nesse caso, a RGLS foi implantada para suprir os requisitos básicos para o monitoramento costeiro sazonal com vistas (SANTOS, 2011): a) a fornecer bases curtas ao método de posicionamento relativo cinemático do GPS, necessárias para se obter precisão compatível com as dimensões das principais feições morfológicas modeladas nas zonas de praia e das variações sedimentares ocorridas na escala de tempo intra anual; b) a prover referencial geodésico unívoco, fixo e relativamente estável no tempo, que permita a repetição dos levantamentos sempre nos mesmos referenciais, a comparação dos resultados de pesquisas realizadas em épocas e áreas diferentes (domínios temporal e espacial), e a conversão para outros tipos de referenciais geodésicos; e c) a permitir a determinação de altitudes ortométricas com alta precisão pelo posicionamento GPS em combinação com modelo geoidal, importante ao monitoramento costeiro por gerar LC e MDE relacionados ao nível médio dos mares e referenciados ao SGB.

Assim, se os estudos realizados em diferentes partes do planeta forem sempre relacionados aos referenciais geodésicos oficiais de seus territórios, tais levantamentos podem ser convertidos para um mesmo referencial geodésico, o que 
permitiria a comparação e integração dos levantamentos de diferentes países, auxiliando em estudos costeiros a nível global. No futuro, a RGLS poderá ser utilizada no monitoramento de deformações, que consiste na comparação das coordenadas e altitudes das estações obtidas em épocas distintas e com um determinado intervalo de tempo. Assim, a primeira campanha de observações consistiu no início dos estudos sobre o monitoramento de deformações na área de estudo, de grande interesse científico devido à intensa dinâmica costeira da área que interfere nas atividades socioeconômicas e no meio ambiente.

\section{AGRADECIMENTOS}

Esta pesquisa foi financiada com recursos de projetos de pesquisa da Rede Cooperativa de Pesquisa Norte-Nordeste no Monitoramento de Áreas sob Influência da Indústria Petrolífera (REDE 05 - PETROMAR, CTPETRO FINEP/PETROBRAS/CNPq): Projetos Cooperativos HIDROSEMA, POTMAR, MOLECO e CRONALOG. As atividades foram desenvolvidas com o apoio logístico do Laboratório de Geoprocessamento do Departamento de Geologia da UFRN (GEOPRO/DG/UFRN) e do Programa de Pós-Graduação em Ciência e Engenharia do Petróleo (PPGCEP). Os autores agradecem especialmente à CAPES, pela concessão de Bolsa de Doutorado ao primeiro autor, e aos revisores anônimos pelas importantes contribuições.

\section{REFERENCIAS BIBLIOGRÁFICAS}

BAPTISTA, P.; BASTOS, L.; BERNARDES, C.; CUNHA, T.; DIAS, J.A. Monitoring Sandy Shores Morphologies by DGPS - A Practical Tool to Generate Digital Elevation Models. Journal of Coastal Research, v.24, n.6, p.1516-1528, 2008.

CHAVES, M.S.; VITAL, H.; SILVEIRA, I.M.; SANTOS, D.A.S. Beach Morphodynamics of the Serra Oil Field (NE, Brazil). Journal of Coastal Research, v. 39, p. 594-597, 2006.

FEATHERSTONE, W.E.; DENTITH, M.C. \& KIRBY, J.F. Strategies for the accurated determination of orthometric heights from GPS. Survey Review, v.34, p.278-295, 1998.

GONÇALVES, R.M.; COELHO, L.S.; KRUEGER, C.P. HECK, B. Modelagem preditiva de Linha de Costa utilizando redes neurais artificiais. Boletim de Ciências Geodésicas, v. 16, n.3, p.420-444, 2010.

GRIGIO, A.M.; SOUTO, M.V.S.; CASTRO, A.F.; AMARO, V.E.; Vital, H.; DIODATO, M.A. Method of analysis of the coastline evolution based in remote sensing and geographical information system products: Guamaré District Rio Grande do Norte - Northeast of Brazil.. Journal of Coastal Research, v. 42, n.2, p.412- 421, 2005.

IBGE (FUNDAÇÃO INSTITUTO BRASILEIRO DE GEOGRAFIA E ESTATÍSTICA) - 2011 - Informações obtidas da página do IBGE na internet: Diosponível em <www.ibge.gov.br> Acesso: 01 de janeiro de 2010. 
KIAMEHR, R.: Optimal fitting of GPS/Levelling and FFT geoid in Iran, NCC Geomatics Conferences, Tehran, Iran, 2002

KOTSAKIS, C. \& SIDERIS, M.G. On the adjustment of combined GPS/levelling/geoid networks. Journal of Geodesy, 73: 412-421, 1999.

LIMA, Z.M.C. Caracterização da dinâmica ambiental da região costeira do Município de Galinhos, Litoral Setentrional do Rio Grande do Norte. 2004. 157f. Tese (Doutorado em Geodinâmica e Geofísica) - Departamento de Geologia, Programa de Pós-Graduação em Geodinâmica e Geofísica, Universidade Federal do Rio Grande do Norte.

MONICO, J.F.G; PÓZ, A.P.D.; GALO, M.; SANTOS, M.C.D; OLIVEIRA, L.C. Acurácia e precisão: revendo os conceitos de forma acurada. Boletim de Ciências Geodésicas. V.15,n.3,p.469-483,2008.

MONICO, J.F.G. Posicionamento pelo GNSS: descrição, fundamentos $e$ aplicações.São Paulo: Editora Unesp, p.477, 2007.

OLLIKAINEN, M. Accuracy of GPS levelling. In: THE XIII GENERAL MEETING OF THE NORDIC GEODETIC COMMISSION. Sweden. Anais 1: p.25-29, 1998.

ROCHA, C.P.; ARAÚJO, T.C.M.; MENDONÇA, F.J.B. Methodology for Location of Shorelines using 3D-GPS Positioning: A Case Study at Sauaçui Beach, Northeast Brazil. Journal of Coastal Research. Jul 2009, v.25, n.4, p.1052-1058, 2009.

SANTOS, M.S.T. Contribuição da Geodésia ao Monitoramento Costeiro do Litoral Setentrional do RN, área de influência da Indústria Petrolífera. 2011. 154f. Tese (Doutorado em Ciência e Engenharia de Petróleo) - Centro de Ciências Exatas e da Terra, Programa de Pós-Graduação em Ciência e Engenharia de Petróleo, Universidade Federal do Rio Grande do Norte, Natal.

SEEBER, G. Satellite Geodesy: Fundations, methods and applications. Walter de Gruyter, N. York, p.531, 1993.

SILVA, D.R.V.; AMARO, V.E.; SOUTO, M.V.S.; NASCIMENTO, M.C.; PEREIRA, B.R.B. Geomorfologia de uma área com alta sensibilidade ambiental na Bacia Potiguar (NE do Brasil). Revista Gestão Costeira Integrada. V.10, n. 4, p.545-566, 2010.

SOUTO, M.V.S.; CASTRO, A.F.; GRIGIO, A.M.; AMARO, V.E.; VITAL, H. Multitemporal analysis of geoenvironmental elements of the coastal dynamics of the region of the Ponta do Tubarão, City of Macau/RN, on the basis of remote sensing products and integration in GIS. Journal of Coastal Research, v.39, p.1618-1621, 2004.

TANAJURA, E.L.X, KRUEGER, C.P. \& GONÇALVES, R.M. Análise da acurácia dos métodos cinemáticos de posicionamento GPS em aplicações costeiras. Boletim de Ciências Geodésicas. v.17, n.1, p.23-36, 2011.

(Recebido em agosto de 2011. Aceito em novembro de 2011). 